\title{
Aneusomy of chromosomes 7 and 17 predicts the recurrence of transitional cell carcinoma of the urinary bladder
}

\author{
A.D. WATTERS, S.A. BALLANTYNE, J.J. GOING*, K.M. GRIGOR† and J.M.S. BARTLETT \\ University Departments of Surgery and *Pathology, Glasgow Royal Infirmary, Glasgow, and †University Department of \\ Pathology, Edinburgh, Scotland
}

Objective To determine if changes in chromosome 7 and 17 copy number can be used to predict recurrence in patients with primary noninvasive (pTa) or superficially invasive (pT1) transitional cell carcinoma (TCC) of the urinary bladder.

Patients and methods Tissue specimens for 129 tumours from 52 patients ( 38 men and 14 women) with pTa/ pT1 TCC at first diagnosis were retrieved from pathology archives. All patient notes were accessed and disease outcome documented for superficial (pTa/ pT1) recurrence or progression to detrusor muscle invasion $(\geqslant \mathrm{pT} 2)$. The tumours were examined for chromosomal copy number of chromosomes 7 and 17 using fluorescence in situ hybridization (FISH) with chromosome-specific probes. The copy number of chromosomes 7 and 17 was determined in interphase nuclei on intact $6 \mu \mathrm{m}$ tissue sections.

Results Aneusomy of chromosomes 7 and 17 was detected in the index primary tumours of 10 of 32 (31\%) patients with subsequent recurrent disease. No aneusomy for these chromosomes was detected in primary tumours from 20 patients with no detectable recurrence $(P=0.0082)$. The relative risk of recurrence was 3.62 times greater (95\% confidence interval 1.6-8.1, Cox's multiple regression $P=0.0019$ ) for patients with chromosomal aneusomy in primary TCC. Neither stage nor grade of the primary tumours was associated with recurrence in these patients, nor was there a significant association with increased grade $(\mathrm{G} 2 / 3)$ or stage $(\geqslant \mathrm{pT} 2)$ at recurrence.

Conclusion These results suggest that the measurement of aneusomy by FISH, using markers for chromosomes 7 and 17, predict recurrence in a subgroup of patients with pTa/pT1 tumours at presentation. This finding may offer a new objective and quantitative test for patients destined to recur.

Keywords Bladder cancer, fluorescence in situ hybridization, chromosomal aneusomy, recurrence, prediction

\section{Introduction}

We have previously reported that monosomy of chromosome 9 predicts recurrence of TCC of the urinary bladder [1]; in the present study, we examined the role of chromosomes 7 and 17 in predicting TCC recurrence. About 13000 new cases of TCC are reported in the UK each year and the high incidence of recurrence makes this the second most prevalent cancer in the UK population. More than $80 \%$ of patients presenting with superficial (pTa/pT1) TCC experience multiple episodes of tumour recurrence [2,3]. Furthermore, in $10-20 \%$ of patients recurrence leads to progression to detrusor muscle invasion [2,3], with a reduction in the 5-year survival rate from $95 \%$ to $35 \%$

Accepted for publication 14 July 1999
$[4,5]$. The unpredictable course of TCC and high risk of recurrence and invasion necessitates intensive monitoring of these patients.

Many genetic lesions have been investigated in TCC without producing tests of diagnostic value [4-13]. However, earlier studies have suggested that trisomy of chromosome 7 may be involved in disease recurrence, whilst polysomy of 17 and loss of heterozygosity (LOH) of $17 \mathrm{p}$ have been strongly associated with increased stage and grade [5,14-17]. This evidence, in conjunction with our previous experience with chromosome 9 [1], led us to propose that the use of these markers in primary tumours might identify patients at risk of recurrence.

Fluorescence in situ hybridization (FISH) has been widely applied to the study of genetic aberrations in TCC [15-17]. It is relatively simple, yields valuable information from intact tissue sections of solid tumours, and allows the relationship between neoplastic and 
non-neoplastic tissue to be studied in situ. In the present study, FISH was used to quantify aneusomy of chromosomes 7 and 17 in TCC, and to identify genetic changes associated with recurrence.

\section{Patients and methods}

Fifty-two patients were selected for this retrospective study, of whom 51 presented with pTa/pT1 TCC (not muscle-invasive). One patient presented with carcinoma in situ (CIS) and subsequently developed TCC. The patients' notes were reviewed and patients allocated to two groups based on their clinical history. Twenty patients with no recurrence (NR) had one superficial (pTa/pT1) carcinoma with no subsequent recurrences at check cystoscopy, and patients with recurrence (REC) presented with several episodes of recurrent TCC after the primary carcinoma, either with no evidence of detrusor muscle-invasive disease (20), or with progression to muscle invasion during the follow-up (11). Patients whose primary tumour or first recurrence was treated by diathermy with no biopsy were excluded. Where available tumour biopsy material was inadequate to allow sectioning for study, the patient was also excluded.

\section{Control and tumour material}

Morphologically normal, disomic urinary bladder epithelium served as a quality control for the FISH method. The primary carcinoma, first recurrence and last documented recurrence (or in patients whose recurrences progressed to pT2 and above, that immediately before and the first muscle-invasive carcinoma) were analysed by FISH. In some patients the muscle-invasive carcinoma was obtained at cystectomy. Tumours were accessed from participating pathology laboratories (Departments of Pathology at Glasgow and Edinburgh Royal Infirmaries) and $6 \mu \mathrm{m}$ serial sections of formalinfixed paraffin wax-embedded sections were cut onto 3aminopropyltriethoxysilane-treated slides. All tumours analysed had a representative section stained with haematoxylin and eosin (H\&E), and were re-staged and re-graded by a specialist urological pathologist (K.M.G.) following the UICC 1978 guidelines [18].

\section{Fluorescence in situ hybridization}

Dual-target FISH was performed with $\alpha$-satellite repeat sequence DNA probes for chromosomes 7 and 17, based on the protocol of Murphy et al. [19]. Briefly the tissue sections were dried at $56^{\circ} \mathrm{C}$, dewaxed, rehydrated and air-dried immediately before use. Pepsin digestion ( $0.4 \%$ pepsin, Sigma, Poole, UK, in $0.2 \mathrm{~mol} / \mathrm{L} \mathrm{HCl}$ ), to reverse the protein cross-linking caused by formalin fixation, was performed at $37^{\circ} \mathrm{C}$ for $45 \mathrm{~min}$ and the pepsin refreshed after $30 \mathrm{~min}$. Sections were washed five times in distilled water and dehydrated. To assess the extent of pepsin digestion, nuclei were stained with $2 \mu \mathrm{g} / \mathrm{mL}$ of 4,6 diamidino-2 phenylindole-2 hydrochloride (DAPI) in anti-fade mounting medium (Vectashield, Vectorlabs, Peterborough, UK). Samples with residual protein masking nuclei (under-digested) were re-digested as appropriate, for a maximum of a further $15 \mathrm{~min}$. Tissue showing loss of nuclear boundaries (over-digestion) was discarded and duplicate slides digested for $30 \mathrm{~min}$. Slides were rinsed in $2 \times \mathrm{SSC}$, where SSC was $0.15 \mathrm{~mol} / \mathrm{L}$ sodium chloride and $0.015 \mathrm{~mol} / \mathrm{L}$ sodium citrate, pH 7.0, and post-fixed in Streck tissue fixative (Alpha Labs, Eastleigh, UK) for $10 \mathrm{~min}$, dehydrated and airdried. Equal volumes of digoxigenin-labelled chromosome 7 and biotin-labelled chromosome 17 (Oncor, Watford, UK) probes diluted 1:10 in hybridization mixture containing $50 \%$ formamide, $2 \times \mathrm{SSC}, 0.5 \mathrm{mg} / \mathrm{mL}$ salmon sperm DNA, $2 \%$ dextran sulphate (all from Sigma), giving a final probe concentration of $10 \mathrm{ng} / \mathrm{mL}$, were applied to the tissue sections on glass coverslips and sealed with rubber cement. Tissue and probe were simultaneously denatured on a Misha unit (Shandon Scientific, Runcorn, UK) for $5 \mathrm{~min}$ at $72^{\circ} \mathrm{C}$ and hybridized overnight at $37^{\circ} \mathrm{C}$. Post-hybridization washes in $50 \%$ formamide, $1 \times$ SSC and $2 \times$ SSC, respectively, were conducted at $42^{\circ} \mathrm{C}$ for $20 \mathrm{~min}$ each. Before immunocytochemical detection, slides were placed in phosphate buffer, pH 8.0, 0.05\% Tween (PNT) and nonspecific tissue binding blocked in PNTB (PNT and 0.5\% Boehringer blocking agent). For two-colour FISH, the first detection layer was sheep antidigoxigenin $(1 \mu \mathrm{g} /$ $\mathrm{mL}$, Boehringer, Lewes, UK); slides were then rinsed in PNT, blocked in $4 \times$ SSC, $0.05 \%$ Tween and $0.5 \%$ blocking agent $(4 \times \mathrm{SSCTB})$. Subsequent detection layers consisted of fluorescein isothiocyanate (FITC) avidin $(25 \mu \mathrm{g} / \mathrm{mL}$, Vectorlabs) and rabbit antisheep Fab fragments (18 $\mu \mathrm{g} / \mathrm{mL}$, Stratech, Luton, UK), biotinylated goat anti-avidin $(5 \mu \mathrm{g} / \mathrm{mL}$, Vector Labs) and Cy3 donkey antirabbit ( $3 \mu \mathrm{g} / \mathrm{mL}$, Stratech), and finally FITC avidin $(25 \mu \mathrm{g} / \mathrm{mL})$ all diluted in $4 \times$ SSCTB. Finally, slides were mounted in Vectashield containing $2 \mu \mathrm{g} / \mathrm{mL}$ DAPI. This protocol yielded results of equal colour intensity for both probes and that were easily quantifiable (Fig. 1).

\section{Evaluation}

The signals were visualized using an epifluorescence microscope (DMLB, Leica, Cambridge, UK), with a 100-W mercury arc lamp, and photomicrographs taken using a Wild 48/52 photoautomat system (Leica). Regions for analysis by FISH were identified by examining H\&Estained slides and areas to be scored marked on an 

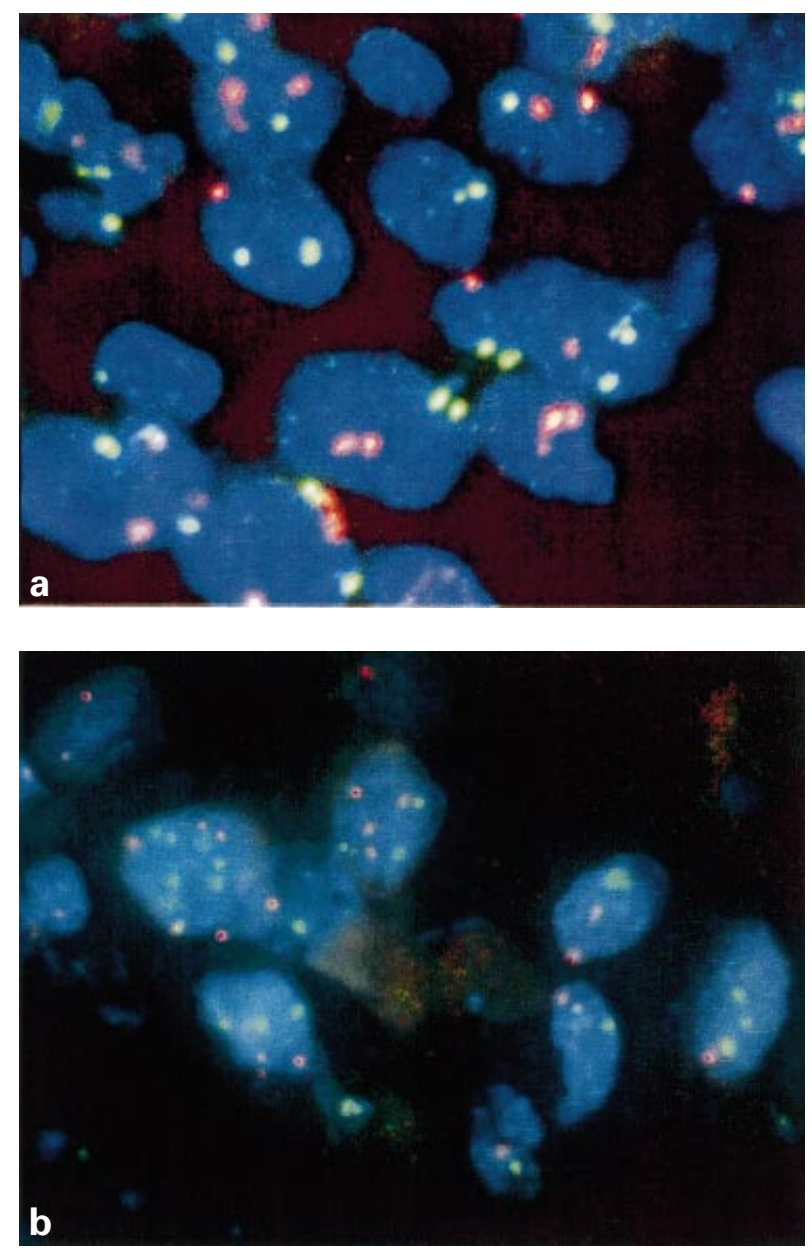

Fig. 1.Photomicrographs of FISH on sections of: A, a disomic bladder tumour. DAPI-stained nuclei (blue fluorescence) show two copies of chromosome 7 (Cy3, red) and chromosome 17 (FITC, green). B, an aneusomic TCC; several copies of chromosomes 7 (red) and 17 (green) represent aneusomy.

enlarged photocopy (200\%) of the section. The number of signals (range 0-8) for each chromosome in at least 200 non-overlapping nuclei in the control (normal urothelium) and carcinoma sections were counted using a multichannel counter and recorded. One to three tumour areas were analysed, a proportion of these by three independent observers.

The mean chromosomal copy number $(\mathrm{MCCN}$, the total number of hybridization signals divided by the total number of nuclei) was used as a measure of overall chromosomal copy number. Monosomy was assessed following the parameters previously defined [1]. Normal ranges for MCCN and monosomy were defined as the mean \pm 3 SD of values for control normal urothelium. Aneusomy was defined as tumours with $\geqslant 10 \%$ of cells with three or more signals per nucleus $[19,20]$. The mean chromosome counts from each tumour area were used to give an overall value for each tumour.
Table 1 The demographics of the patients with no recurrence (NR) or recurrence (REC). One patient's primary event was reported as CIS and another as dysplasia. There were no significant differences in any category between the groups

\begin{tabular}{lll}
\hline Group & NR & REC \\
\hline Number & 20 & 32 \\
Mean (SD) age at diagnosis, years & $67.39(8.87)$ & $68.06(11.36)$ \\
Sex (M : F) & $16: 4$ & $22: 10$ \\
Median (range) follow-up (months) & $50.0(7-151)$ & $45.5(2-151)$ \\
Median (range) no. of cystoscopies & $6.5(1-14)$ & $6.0(1-27)$ \\
Stage at diagnosis, n (\%) & & \\
pTa & $14(70)$ & $18(56)$ \\
pT1 & $5(25)$ & $12(38)$ \\
pT2/2+ & $1(5)$ & 0 \\
Grade at diagnosis & & \\
1 & $6(30)$ & $12(38)$ \\
2 & $12(60)$ & $13(41)$ \\
3 & $2(10)$ & $5(16)$ \\
\hline
\end{tabular}

Mann-Whitney tests were used to compare differences in the number of cystoscopies and length of follow-up between patient groups, and ANOVA to compare age at diagnosis. The chi-squared and Fisher's exact tests (where expected counts were $<5$ ) were used to compare stage and grade at diagnosis, and sex ratios between patient groups, and to compare aneusomy/normosomy and with stage and grade. Kaplan-Meier regression analyses were used to predict recurrence. Cox's multiple regression analysis was applied to determine the significance of stage, grade and aneusomy in predicting recurrence.

\section{Results}

For primary tumours, there were no significant differences in $\mathrm{M}$ : F ratios $(P=0.21)$, mean age at diagnosis $(P=0.83)$, length of follow-up $(P=0.53)$ and number of cystoscopies $(P=0.95)$, nor were there significant differences in stage $(P=0.17)$ and grade $(P=0.18)$ between the patient groups (Table 1). One patient in the NR group was re-staged by the reference pathologist as a pT2 tumour. One patient's primary event in the REC group was reported as a CIS, another as dysplasia. A further 77 tumours were analysed from patients in the REC group, of which 52 (68\%) were pTa/pT1, 21 (27\%) were $\geqslant$ pT2, $27(35 \%)$ were $\mathrm{G} 1$ and $46(60 \%)$ were $\mathrm{G} 2$ or G3. Two tumours were classed at pTxGx, one as dysplastic and one as CIS.

\section{Fluorescent in situ hybridization}

Areas from 42 tumours were assessed by three independent observers ( 35 by observers $\mathrm{A}$ and $\mathrm{B}$, and 
seven by observers $\mathrm{A}$ and $\mathrm{C}$ ) to determine the reproducibility of the results; there was close concordance among observers. The mean inter-observer variation was $6.7 \pm 0.6 \%$ for chromosome 7 and $7.3 \pm 0.8 \%$ for chromosome 17 copy numbers.

The MCCN was $1.63 \pm 0.07$ and $1.61 \pm 0.08$ for chromosomes 7 and 17, respectively. Values of $<1.42$ and $<1.37$ were taken to indicate monosomy for chromosomes 7 and 17, respectively, and those $>1.84$ and $>1.85$, respectively, were defined as aneusomic for 7 and 17. Because of the nuclear truncation inherent in thin histology sections, disomic tumours had MCCN values of $<2$.

Fifty-two primary carcinomas from the two groups of patients were analysed for abnormalities of either chromosomes 7 or 17. Eighteen tumours were G1, of which two were aneusomic, and 32 tumours were G2/3, of which seven were aneusomic $(P=0.68)$. Twenty-eight pTa tumours were normosomic and four aneusomic, and 13 pT1 were normosomic whilst five were aneusomic $(P=0.44)$. One primary tumour reported as dysplasia was also aneusomic. The primary event reported as CIS was normosomic for both chromosomes.

Ten of 32 primary (first) tumours (31\%) from the REC group and none of 20 from the NR group had abnormalities of chromosomes 7 and $17(P=0.0082)$. Neither stage $(P=0.48)$ nor grade $(P=0.98)$ of the primary tumour were associated with recurrence in the REC group. By univariate analysis, patients whose primary tumours showed aneusomy of 7 or 17 had significantly shorter disease-free intervals than in patients with no chromosomal abnormalities $(P<0.001)$. On multivariate analysis, recurrence could be predicted by aneusomy and patients with abnormalities of 7 and/or 17 were 3.62 times more likely to recur (95\% CI, 1.6-8.1; Cox's multiple regression, $P=0.0019)$ than patients with normal copy number for chromosomes 7 and 17 (Fig. 2).

\section{Recurrent tumours}

A further 77 tumours were assessed for abnormalities in the REC group. Of patients with aneusomy in their primary (index) tumours, $60 \%$ remained aneusomic in all subsequent tumour events studied. Two patients were aneusomic in one tumour recurrence only. Following recurrence, a further three patients had chromosomal abnormalities which were then consistently recapitulated in all subsequent tumours analysed from these patients. A change in grade or stage in subsequent recurrences was not associated with aneusomy in the primary tumour. Four of 10 patients with aneusomy in their primary tumours developed a change in grade, to Grade 2 or $3(P=0.28)$; four of 10 developed a change in stage, to pT2 and above $(P=0.42)$. Of 22 normosomic tumours, $12(55 \%)$ had increased stage and seven $(32 \%)$ increased grade.

Comparing aneusomy with stage and grade in all tumours analysed, overall aneusomy for either chromosome 7 or 17 or both were detected in four of $47(8.5 \%)$ G1 and 37 of $76(49 \%)$ G2/3 tumours $(P<0.001)$, including both primary and recurrent tumours. Similarly 20 of $101(20 \%)$ pTa/pT1 tumours and 14 of $22(64 \%)$ pT2+ tumours had aneusomy for either or both chromosome markers $(P<0.001)$.

\section{Discussion}

It could be argued that recurrence is by far the most significant event in the clinical management of bladder cancer. The high frequency of recurrence and the associated increase in risk of progression in patients presenting with superficial TCC is the underlying reason for the extremely intensive follow-up required for patients with TCC. Thus far, predictive markers of recurrence have not been available. The ability to predict recurrence would undoubtedly improve treatment options in this extremely prevalent cancer.

Despite extensive research into genetic alterations in bladder cancer, and detailed models which link such changes to tumour initiation and progression, there has been no concurrent improvement in patient management as a result of these investigations. This may partly be because there has been no investigation of recurrence as a distinct genetically driven event in bladder cancer.

Aneusomy for chromosomes 7 and 17, as detected by FISH in the present study in primary TCCs, only occurred

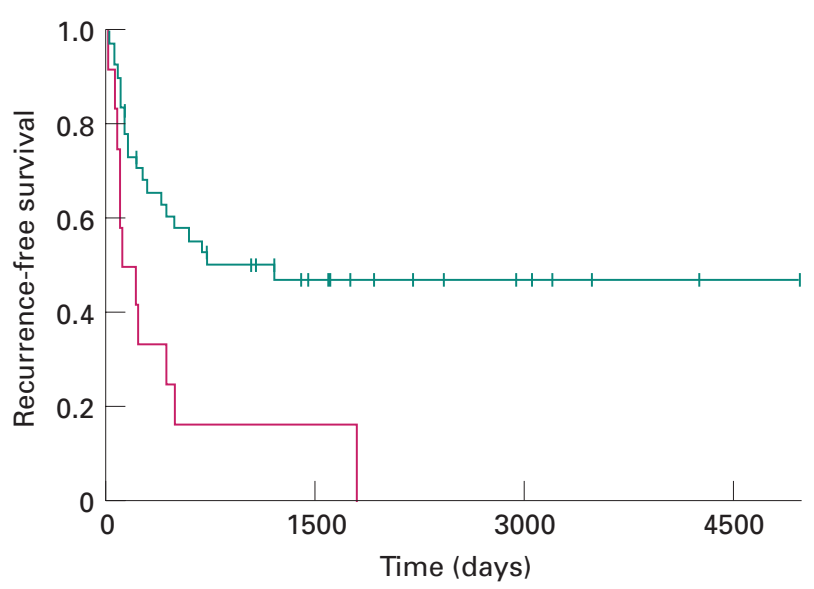

Fig. 2. The time to recurrence, showing the recurrence-free intervals for patients with normosomy for both chromosomes 7 and 17 (green) and aneusomy chromosome 7 and/or 17 detected in primary tumours (red). Vertical lines represent the extent of followup. The relative risk of recurrence (Cox's multiple regression analysis) was 3.62 times greater for patients with abnormal chromosome numbers $(P=0.0019)$. 
in patients who subsequently developed recurrence. Aneusomy in primary tumours did not predict an increase in grade or stage at subsequent recurrence. Ten of 32 (31\%) patients had aneusomy in their primary tumours. There appears to be a strong association between aneusomy of these chromosomes and early recurrent disease (odds ratio 3.62, Cox's multiple regression), independent of the known effects of high (pT1) stage and grade (G2/G3) in predicting recurrence. This is the second chromosomal marker to be associated with disease recurrence. Bartlett et al. [1] showed that monosomy of chromosome 9 in primary tumours from a similar patient cohort is strongly predictive of disease recurrence in TCC patients. About 35\% of patients in their study were monosomic for chromosome 9, of whom 91\% developed disease recurrence. The possibility that these markers identify independent genetic routes to recurrence may allow the identification of the risk of recurrence in up to two-thirds of patients presenting with superficial bladder cancer.

At present, frequent cystoscopy is the only means by which patients can be accurately monitored. This combined with transurethral resection and, in appropriate cases, intravesical chemotherapy, will reduce the risk of progression to muscle invasion [21]. It has been suggested that the ability to predict recurrence at presentation could improve treatment and follow-up of patients by reducing rates of recurrence by $30 \%$ over 5 years [22], but at present it is not possible to accurately identify patients at high risk of recurrence, and therefore progression, by cystoscopy and pathology alone. The use of a diagnostic marker of high risk of recurrence would improve the treatment and the quality of life in a significant proportion of patients. This would reduce the need for frequent attendance at clinic, thereby minimizing the patients' stress and anxiety. FISH is an accurate, reproducible and quality-controlled system for identifying markers of recurrence in TCC. Intervention in patients at high risk of recurrence because of tumour aneusomy, using single or multiple doses of epirubicin, mitomycin $\mathrm{C}$ or intravesical BCG, could significantly reduce recurrence rates in the population with bladder cancer in the UK.

Neither stage nor grade can be used in a similar way to predict recurrence. Whilst both increased stage and grade are associated, both in this study and elsewhere, with increasing genetic abnormalities, it is striking that in the primary (first) tumours analysed here, aneusomy predicted recurrence independently of either grade or stage. The significant association between aneusomy and $\geqslant$ pT2 in all the tumours studied, rather than $\mathrm{pTa} / 1$ disease, would not compromise this finding as patients with detrusor muscle invasion ( $\geqslant$ pT2) are not routinely left untreated. The association of aneusomy with high grade supports the clinical observation that high-grade tumours are more likely to recur, and may provide a means of identifying those patients with higher grade disease at greatest risk of recurrence.

The association between aneusomy of chromosomes 7 and 17 in patients at high risk of subsequent recurrence indicates that genes on these chromosomes promote recurrence. Changes in p53 expression and subchromosomal alterations of chromosome 17 have been linked with CIS and progression to detrusor muscle invasion by papillary TCC. In contrast, relatively little is known about genetic alterations on chromosome 7 and outcome in TCC. However, studies by Neal et al. [23] have shown that overexpression of EGFR is a risk factor for recurrence in TCC. This could be linked to increased copy number of this gene, which is on chromosome 7 . This event may also be associated with genetic instability [14]. Low-level amplification of c-erbB2, on chromosome 17, may also promote recurrence, with subsequent loss of p 53 driving progression events. TP53 mutations are known to destabilize the genome [4,6] which would allow cells to gain a more malignant phenotype. Previous reports have found that both overexpression of c-erbB2 and abnormal expression of p53 are associated with progression [5-7]. Saran et al. [6] reported that overexpression of EGFR was associated with high-grade tumours, and Ozen [5] reported that there was a strong association with high stage and grade. Ozen [5] suggested that c-erbB2 and TP53 could be responsible for progression to high grade and lamina propria invasion. These studies and others $[4,14,24]$ support the findings of abnormalities in 7 and 17 reported here. However, there have been no studies associating such changes specifically with recurrence and it may be dangerous to extrapolate alterations associated with progression to a separate disease event.

There was a striking concordance in the copy number of chromosomes 7 and 17 throughout this study, as has been previously shown for renal cancers [20]. Separate investigations have shown that these changes occur independently of polyploidy and can therefore be regarded as chromosome-specific events [25].

In conclusion, the present results strongly support the role for genetic analysis in the predictive diagnosis of disease recurrence in patients with TCC. Such diagnosis offers significant opportunities for treatment intervention and improvement of patient outcome. Aneusomy of chromosomes 7 and 17 , occurring in $31 \%$ of the present patients with $\mathrm{pTa} / \mathrm{pT} 1$ disease at presentation, predicted recurrence.

\section{Acknowledgements}

We thank Dr W Angerson for statistical help. This research was supported by a Scottish Office Home and Health Department Grant number SHHD C2263. 


\section{References}

1 Bartlett JMS, Watters AD, Ballantyne SA, Going JJ, Grigor KM, Cooke TG. Quantitative FISH. Is chromosome 9 loss a marker of disease recurrence in TCC of the bladder? $\mathrm{Br} J$ Cancer 1998; 77: 2193-8

2 Anonymous. Office of Population Censuses and Studies 1969-87; Office of Population Censuses and Studies, DH1, Cancer Statistics, HMSO, 1969-1987

3 Anonymous. Office of Population Censuses and Studies 1971-84; Office of Population Censuses and Studies, MB1, Cancer Statistics, HMSO, 1971-1984

4 Knowles MA. Molecular genetics of bladder cancer. Br J Urol 1995; 75: 57-66

5 Ozen H. Transitional cell carcinoma of the bladder. Curr Opin Oncol 1996; 8: 259-63

6 Saran KK, Gould D, Godec CJ, Verma. RS. Genetics of bladder cancer. J Mol Med 1996; 74: 441-5

7 Underwood M, Bartlett J, Reeves J, Gardiner S, Scott R, Cooke T. cerbB2 gene amplification: a molecular marker in recurrent bladder cancers? Cancer Res 1995; 55: 2422-30

8 McCredie M. Bladder and kidney cancers. Cancer Surveys 1994; 19-20: 343-68

9 Cordon-Cardo C, Sheinfeld J. Molecular and immunopathology studies of oncogenes and tumor-suppressor genes in bladder cancer. World J Urol 1997; 15: 112-9

10 Sandberg AA, Berger CS. Review of chromosome studies in urological tumours. II. Cytogenetics and molecular genetics of bladder cancer. J Urol 1994; 151: 545-60

11 Povey S, Attwood J, Chadwick B et al. Report on the Fifth international workshop on chromosome 9. Ann Human Genetics 1997; 61: 83-206

12 Sauter G, Moch H, Carroll P, Kerschmann R, Mihatsch MJ, Waldman FM. Chromosome-9 loss detected by fluorescence in situ hybridization in bladder cancer. Int J Cancer 1995; 64: 99-103

13 Kallioniemi A, Kallioniemi OP, Citro G et al. Identification of gains and losses of DNA sequences in primary bladder cancer by comparative genomic hybridization. Genes Chromosomes Cancer 1995; 12: 213-9

14 Waldman FM, Carroll PR, Kerschmann R, Cohen MB, Field FG, Mayall BH. Centromeric copy number of chromosome 7 is strongly correlated with tumour grade and labelling index in human bladder cancer. Cancer Res 1991; 51: 3807-13

15 Sauter G, Deng G, Moch H et al. Physical deletion of the p53 gene in bladder cancer. Detection by fluorescence in situ hybridization. Am J Pathol 1994; 144: 756-66

16 Pycha A, Mian C, Haitel A, Hofbauer J, Wiene H, Marberger M. Fluorescence in situ hybridisation identifies more aggressive types of primary non invasive (stage pTa) bladder cancer. J Urol 1997; 157: 2116-9

17 Sauter G, Moch H, Gasser TC, Mihatsch MJ, Waldman FM. Heterogeneity of chromosome 17 and erbB-2 gene copy number in primary and metastatic bladder cancer. Cytometry 1995; 21: 40-6

18 UICC TNM classification of malignant tumours. Geneva: UICC, 1978

19 Murphy DS, McHardy P, Coutts J et al. Interphase cytogenetic analysis of erbB2 and topoII $\mu$ co-amplification in invasive breast cancer and polysomy of chromosome 17 in ductal carcinoma in situ. Int J Cancer 1995; 64: 18-26

20 Corless CL, Aburatani H, Fletcher JA, Houseman DE, Amin MB, Weinberg DS. Papillary renal cell carcinoma quantitation of chromosomes 7 and 17 by FISH, analysis of chromosome $3 \mathrm{p}$ for $\mathrm{LOH}$, and DNA ploidy. Diag Mol Pathol 1996; 5: 53-64

21 Foresman WH, Messing EM. Bladder cancer: natural history, tumor markers and early detection strategies. Semin Surg Oncol 1997; 13: 299-306

22 Tolley DA, Parmar MKB, Grigor KM et al. The effect of intravesical mitomycin $\mathrm{C}$ on recurrence of newly diagnosed superficial bladder cancer: a further report with 7 years follow-up. J Urol 1996; 155: 1233-8

23 Qureshi KN, Lunec J, Neal DE. Molecular-biological changes in bladder cancer. Cancer Surveys 1998; 31: 77-98

24 Yokogi H, Wada Y, Moriyama-Gonda N, Igawa M, Ishibe T. Genomic heterogeneity in bladder cancer as detected by fluorescence in situ hybridisation. Br J Urol 1996; 78: 699703

25 Bartlett JMS, Watters AD, Adie L, Going JJ, Grigor KM. Chromosome aberrations in TCC predictive of disease outcome are independent events not associated with polyploidy. British Association for Cancer Research, Association of Cancer Physicians and Royal Society of Medicine (Oncology section): Joint Winter meeting 1997. Br J Cancer 1998; 78: 141-58

\section{Author}

A.D. Watters, BSc, Research Assistant.

S.A. Ballantyne, MD.

J.J. Going, PhD, MD, Consultant Pathologist.

K.M. Grigor, MD, Consultant Pathologist.

J.M.S. Bartlett, PhD, Senior Lecturer.

Correspondence: Mrs A.D. Watters, University Department of Surgery, Level 2, Queen Elizabeth Building, Glasgow Royal Infirmary, Alexandra Parade, Glasgow G31 2ER, UK. 